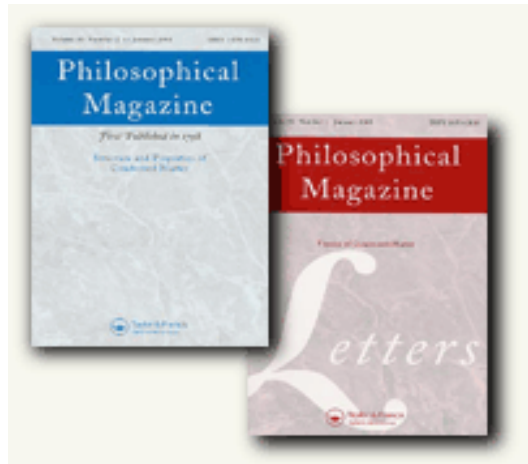

\title{
Mechanical analysis of indentation experiments with conical indenter
}

\begin{tabular}{|r|l|}
\hline Journal: & Philosophical Magazine \& Philosophical Magazine Letters \\
\hline Manuscript ID: & TPHM-05-Nov-0489.R2 \\
\hline Journal Selection: & Philosophical Magazine \\
\hline Date Submitted by the \\
Author: & $12-J a n-2006$ \\
\hline Complete List of Authors: & $\begin{array}{l}\text { Felder, Eric; Ecole des Mines de Paris } \\
\text { Ramond-Angélélis, Céline; Ecole des Mines de Paris }\end{array}$ \\
\hline Keywords: & indentation, computer modelling \\
\hline Keywords (user supplied): & cone, pyramid \\
\hline
\end{tabular}

\section{scholarONE" \\ Manuscript Central}




\title{
Mechanical analysis of indentation experiments with conical indenter
}

\author{
E. Felder and C. Ramond-Angélélis \\ Centre de Mise en Forme des Matériaux (CEMEF), UMR 7635 CNRS-Ecole des Mines de Paris \\ BP 207 F 06904 Sophia-Antipolis France ${ }^{l}$
}

\begin{abstract}
We study using the finite element method the pyramidal indentation performed on elastic-perfecly plastic (EPP) solids: their effective elastic modulus $E^{*}$ to the flow stress $\sigma_{0}$ ratio ranges from 2.79 (quasi-elastic solid) to 2790 (quasi rigid-perfecly plastic (RPP) solid). The friction shear stress was taken equal to zero or its maximal value. First we analyse the two-dimensional indentation with a rigid axisymmetric cone (semiapical angle $\theta=703 \mathrm{deg}$ ). We provide the evolution with the indentation index $X=\left(E^{*} / \sigma_{0}\right) \cot \theta$ of the indent profile, the shape ratio $c=h_{c} / h$, where $h\left(h_{c}\right)$ is the indentation (contact) depth, and the hardness $H$. The influence of friction becomes significant for $X>10$. We validate our results by comparison with the results related to RPP solid and the results of three-dimensional numerical simulation of the Vickers and Berkovich pyramidal indentation for $X=1,30$ and 100. A method for interpreting the results of instrumented indentations is proposed and compared with the Oliver and Pharr method.
\end{abstract}

\section{$\S 1$. INTRODUCTION}

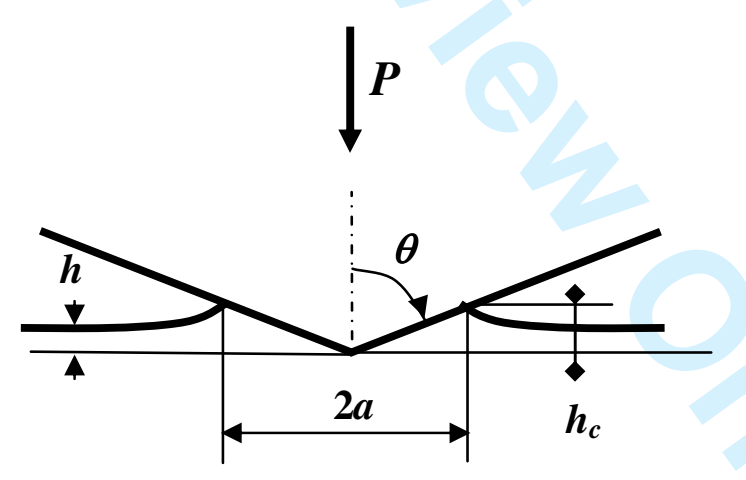

Figure 1. Conical indentation and the related main quantities: penetration depth $h$, contact depth $h_{c}$, contact radius $a$, indenter angle $\theta$ and indentation force $P$.

The indentation experiments [1] and especially instrumented indentations performed with the Berkovich pyramid [2] are very commonly used. But their interpretation remains a difficult problem. The mechanical analysis of indentation provides two very important quantities for the instrumented indentation [2]:

\footnotetext{
${ }^{1}$ Tél. : (33) (0)4 93957428 Fax : (33) (0)4 92389752 E.Mail : Eric.Felder@ensmp.fr
} 


$$
\text { the shape ratio } c=\frac{h_{c}}{h} \quad \text { the reduced hardness } H^{*}=\frac{H}{\sigma_{0}}
$$

The shape ratio $c$ provides the contact depth $h_{c}$ (figure 1) starting from the indentation depth $h$, and so the projected contact area $A=24.5 c^{2} h^{2}$ (for a perfect pyramid) and the hardness $H=P / A$, the reduced hardness provides the link between the hardness and the flow stress $\sigma_{0}$ of the material.

A complete description of the axisymmetric indentation of elastic solids (Young's modulus $E$, Poisson ratio $v$ ) is available [3]: for conical indenter $c=2 / \pi$ and $H^{*}=X / 2$ (see below). The conical indentation of rigid-perfectly plastic (RPP) solid has been analysed mainly by the slip line field (SLF) method [4]. For elastic-perfectly plastic (EPP) solids Johnson [5] provides the matter balance in the model of the expansion of a spherical cavity proposed by Hill and so estimates $H^{*}$ versus the indentation index $X$ :

$$
X=\frac{E^{*}}{\sigma_{0}} \cot \theta
$$

$E^{*}$ is the effective elastic modulus [2,3] (for rigid indenter $E^{*}=E /\left[1-v^{2}\right]$ ); $2 \theta$ is the cone apical angle. But according to this model whose velocity field is purely radial, the sample surface remains plane (the shape ratio $c=1$ ). By extending the elastic analysis Oliver and Pharr [2] proposed a relation to estimate the shape ratio starting from the initial unloading slope or contact stiffness $S=d P / d h$. By defining a reduced contact stiffness $m_{d}$, their relation is:

$$
c=1-\frac{0.75}{m_{d}} \quad \text { with } \quad m_{d}=\frac{h}{P} \frac{d P}{d h}
$$

Another relation has been proposed later by Bec et al. [6]:

$$
c=1.2\left(1-\frac{1}{m_{d}}\right)
$$

where the factor 1.2 has been deduced from the observation of the residual pile-up induced by indentation of a gold film. More recently Dao et al [7] and Bucaille et al. [8] proposed alternative method for the interpretation of instrumented indentation based on the value of the Kickc's constant $C=P / h^{2}$ and numerical simulations of the conical indentation of materials with the rheological behaviour of metals. Despite these works, the relation (3) (named below O\&P relation) remains the most often used relation despite the fact that it predicts always sink-in (shape ratio $c<1$ ) and so is certainly not suitable for workhardened metals where pileup occurs (the shape ratio $c>1$ ) $[1,8,9]$.

The aim of this paper is to present the results of numerical simulations with the finite element method of the conical indentation of EPP solids [10]. A detailed description of the characteristics and applications of the industrial computer code used in this study (Forge $2^{\circledR}$, Forge $3^{\circledR}$ ) is available online [11].The main work concerns the influence of $E^{*} / \sigma_{0}$ and friction on the indentation with the cone equivalent to the Vickers and Berkovich pyramids $(\theta=70.3$ deg). We compare the results with elastic and SLF analysis and the results of some numerical simulations of Vickers and Berkovich indentations. 


\section{$\S 2$. CONDITIONS OF THE NUMERICAL MODELLING}

The axisymmetric indentation is modelled with Forge $2^{\circledR}$, a two dimensional axisymmetric implicit finite element code which is able to simulate large material displacements and deformations. A two-dimensional rectangular mesh incorporating sixnodes elements is constructed. Elements have a length of $0.04 h_{\max }$ near the indenter and of $3 h_{\max }$ far from the indenter. The size of the domain was chosen so that the boundary conditions have no influence on the results. The indenter is rigid and is modelled as an axisymmetric cone with a semiapical angle $\theta=70.3 \mathrm{deg}$.

Pyramidal indentation is modelled with Forge $3^{\circledR}$ implicit code whose performances are very similar to those of Forge $2^{\circledR}$. For symmetry reasons the domain is $1 / 8^{\text {th }}$ (Vickers pyramid) or $1 / 6^{\text {th }}$ (Berkovich pyramid) of a right-angled parallelepiped. The indenter is rigid. Elements of the domain are three-dimensional meshes with four-node tetrahedra. Far from the indenter, elements have a typical length of about $h_{\max }$. With the Forge ${ }^{\circledR}{ }^{\circledR}$ software, parallepiped boxes are used, and where the mesh is refined, 20 nodes are at least in contact with the indenter. More details concerning simulation of the scratch test and indentation test are given in [6].

The materials are homogeneous and isotropic. The inertial forces are assumed negligible. At each time the strain rate tensor is the sum of an elastic strain rate tensor and a plastic strain rate tensor (elastoplastic material):

$$
\dot{\varepsilon}=\dot{\varepsilon}^{e l}+\dot{\varepsilon}^{p l}
$$

The elastic behaviour is modelled by the classical linear law with two parameters: Poisson's ratio, $v=0.3$, and Young's modulus, $E$. The yield condition is given by the von Mises yield criterion with the flow stress $\sigma_{0}$ and the associated flow law. In 2D simulations the effective elastic modulus $E^{*}$ to the flow stress $\sigma_{0}$ ratio ranges from 2.79 to 2790 ; so the indentation index $X$ ranges from 1 to 1000 for $\theta=70.3 \mathrm{deg}$. In 3D simulations we restrict the calculations to $X=1,30$ and 100 . Friction is characterised by the Tresca's friction coefficient $\bar{m}$ which defines the friction shear stress according to the relation:

$$
\tau=\bar{m} \frac{\sigma_{0}}{\sqrt{3}} \quad 0 \leq \bar{m} \leq 1
$$

This friction law is commonly used to model friction in metal forming analysis [12], it is equivalent to the Coulomb's law if the contact pressure is uniform. In 2D simulations we compare the results for $\bar{m}=0$ (zero friction) and $\bar{m}=1$ (maximal friction because the friction shear stress is equal to the maximal shear stress of the material according to the yield criterion). Because our aim is to estimate the reliability of the 2D approach and because the $3 \mathrm{D}$ simulations are very time consuming, in $3 \mathrm{D}$ simulations we consider only the zero friction case.

\section{§ 3. RESULTS OF THE AXISYMMETRIC APPROACH}

\section{$\S 3.1$. Curves $P$-h and nature of the results}

Because the material is homogeneous and the cone is almost perfect, the reduced force-displacement curve $P^{*}-h *$ where the force and the displacement are divided by their maximal values does not depend on the maximal value of $h$. In addition, the friction for this high indenter angle has very small influence on it as reported in other previously published works [8,13]. Figure 2 provides this curve for $X=5$ and $X=1000$. Clearly the case $X=5$ 
corresponds to a highly elastic material with a large recovery; on the contrary, the curve for $X=1000$ corresponds to an almost rigid-perfectly plastic (RPP) material with very small recovery.

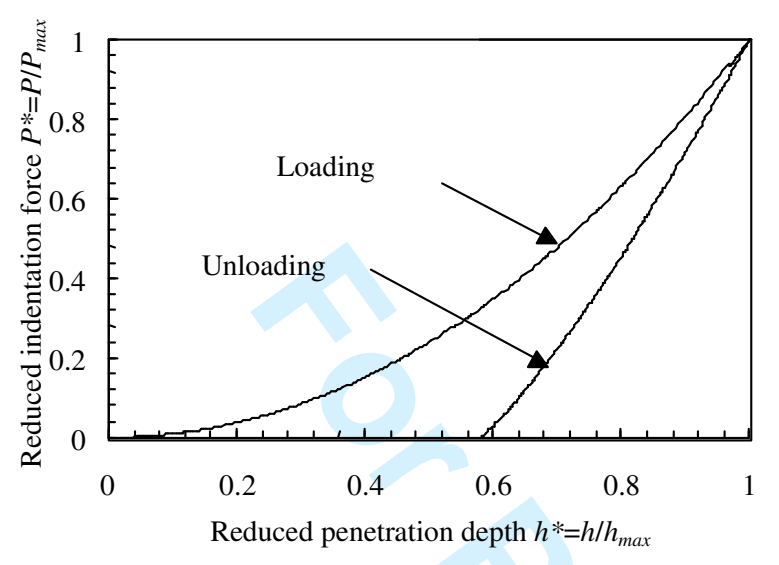

a) $X=5$

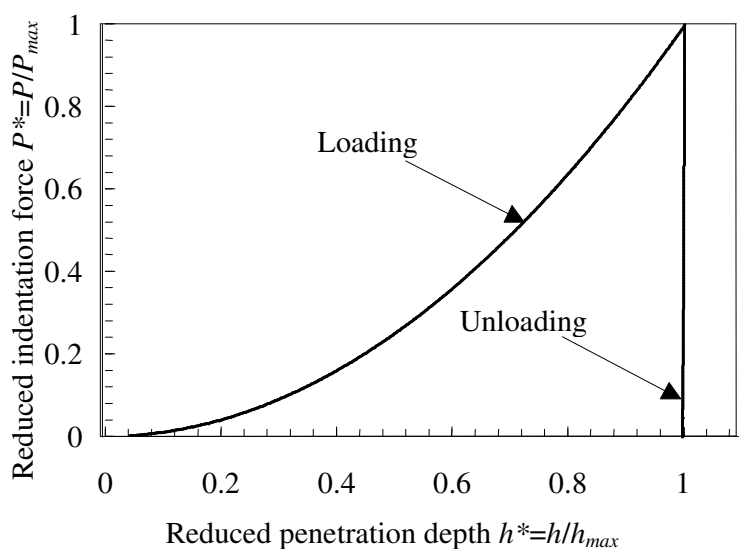

b) $X=1000$

Figure 2. Influence of the indentation index $X$ on the reduced force-displacement $P^{*}-h^{*}$ curve (the curves $P$ - $h$ do not vary significantly with friction).

The loading curve is simply $P=C h^{2}$ (Kick's law). We observe that the unloading curve can be described with a very good approximation by a power function:

$$
\text { Unloading } P \approx B\left(h-h_{f}\right)^{m}
$$

So by applying the procedure of a power curve fit by the least square method proposed by Oliver and Pharr [2] it was possible to estimate accurately the exponent $m$ of the unloading curve, the reduced recovery depth $\Delta h^{*}=1-h_{f} / h_{\max }$, where $h_{f}$ is the residual indentation depth, and the contact stiffness $S$. Due to the geometrical similarity, the various quantities $c, H^{*}, m_{d}$, $m$ and $\Delta h^{*}$ do not depend on the maximal value of the force $P$ or the indentation depth $h$. The evolution versus $X$ of $c, H^{*}, m_{d}$ has been fitted by polynomial curve for zero friction and maximal friction (cf. Appendix).

\section{$\S 3.2$. Contact geometry}

The indent profiles increase in direct relation with $h_{\max }$. So figure 3 provides for zero friction and for the various values of the indentation index the reduced profiles (the dimensions are divided by the maximal penetration depth $h_{\max }$ ) under load and after unloading:

- Under load the material sinks in for $X<30(c<1)$ and piles up for $X>30(c>1)$.

- A very important result of these calculations is that elasticity has some influence in the whole range of values of the indentation index because the indentation profile is not yet constant between $X=200$ and 1000. Even for $X=1000$, where the shape ratio $c \sim 1.25$ we observe some slight elastic recovery during unloading.

- After unloading we observe in all cases an indent with a pile-up. If the indent is not very marked for the quasi-elastic case $X=1$ and even $X=5$, the pile-up is apparent for $X \geq 10$; the radial distance between the indentation axis and the residual bulge top $a$ ' is related to the contact radius $a$ under load by $a^{\prime} / a=1+\delta(X)$ where $\delta(X)$ is a decreasing function of $X$ which tends toward 0 ; in addition $\delta(X)<10 \%$ for $X \geq 10$ : so the 
measurement of the residual indent radius $a$ ' provides an under-estimation of the true indentation pressure $H=P /\left(\pi a^{2}\right)$ with an error lower than $20 \%$. This validates the Meyer procedure where the hardness is deduced from the residual indent [1]. As we see later that these profiles depend on friction for $X>10$.

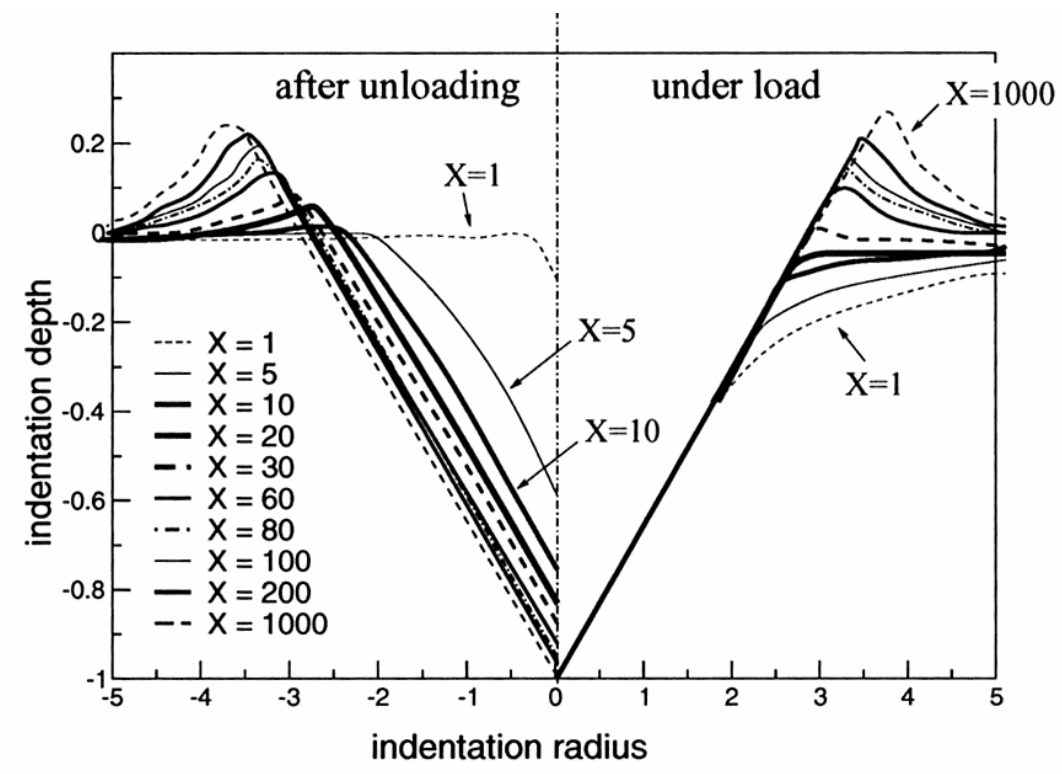

Figure 3. Evolution versus the indentation index $X$ of the indent profile under load and after unloading (70.3 deg cone, zero friction). The coordinates are divided by the indentation depth.

We see in figure 4 that the shape ratio $c$ increases steadily with the indentation index $X$ :

- For $X \leq 10$, the shape ratio does not depend on friction and increases as the logarithm of $X$, from the elastic value $0.63(\sim 2 / \pi)$ for $X=1$ to 0.84 for $X=10$.

- For $X>10$, its increase is slower, but always significant and $\mathrm{c}$ is lower if the friction increases: For $X=1000$ which corresponds to an almost RPP solid (see $\S 4.1$ ), when the friction coefficient increases from 0 to 1 , the shape ratio decreases from 1.25 to 1.16.

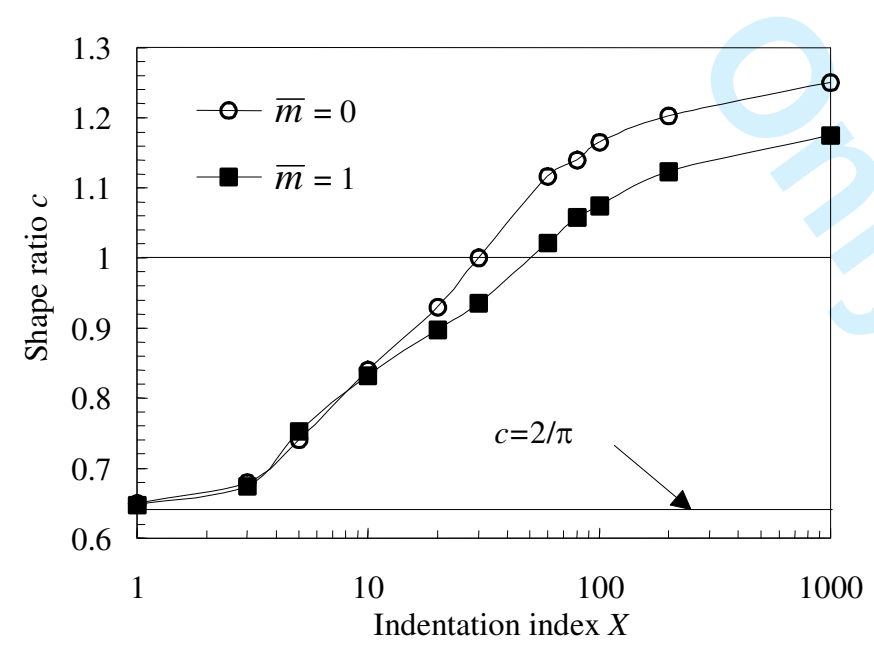

Figure 4. Evolution of the shape ratio $c$ for zero friction and maximal friction (70.3 deg cone) versus the indentation index $X$. 


\section{§ 3.3. Hardness and unloading characteristics}

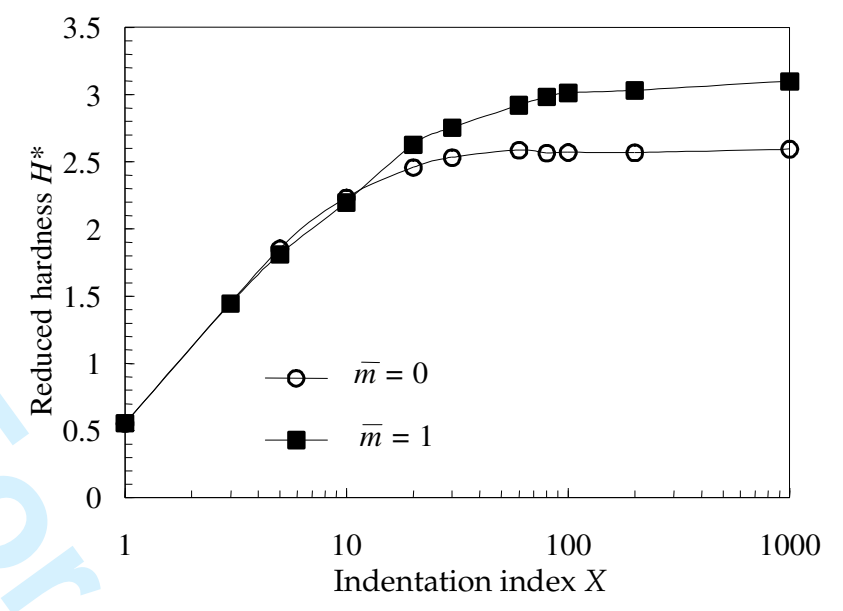

Figure 5. Evolution of the reduced hardness $H^{*}=H / \sigma_{0}$ for zero friction and maximal friction (70.3 deg cone) versus the indentation index $X$.

As reported previously, friction has only a very small influence on the force-displacement $P$ - $h$ curve, but because it can have a significant influence on contact geometry (figure 4), friction influences the value of the hardness. The reduced hardness $H^{*}$ increases with $X$, but this increase comprises two main steps (figure 5):

- The initial increase for $1 \leq X \leq 10$, where $H$ is smaller than $2.24 \sigma_{0}$, is logarithmical and does not depend on friction; it is in agreement with the model of the expansion of the spherical cavity [5].

- For $X>10$, the increase is slower and the hardness increases with friction: for zero friction hardness tends toward $2.6 \sigma_{0}$, and is almost constant for $X>30$; on the contrary at the maximal friction the hardness remains always an increasing function of $X$ and attains the value $3.12 \sigma_{0}$ for $X=1000$.

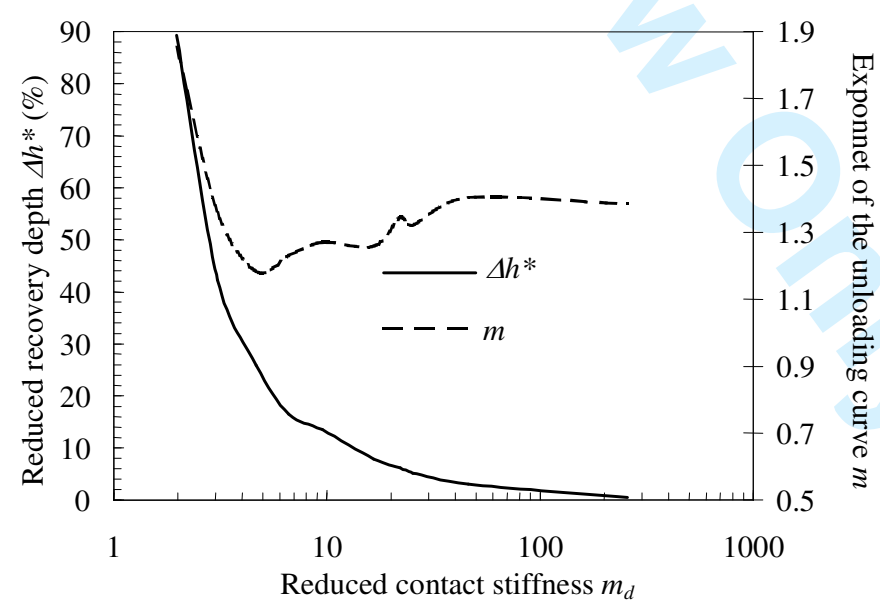

Figure 6. Evolution of the reduced recovery depth $\Delta h *$ and the exponent $m$ of the unloading curve for zero friction (70.3 deg cone) versus the reduced contact stiffness $m_{d}$.

The reduced contact stiffness $m_{d}$ is an increasing function of $X$ varying from about 2 for $X=1$ to about 300 for $X=1000$ (cf. Appendix). The reduced recovery depth $\Delta h^{*}$ decreases steadily as $m_{d}$ increases and falls to very low values for $X=1000$ (Figure 6). On the contrary, the exponent $m$ of the 


\begin{tabular}{cllllll}
\hline Contact & \multicolumn{2}{c}{ Present results $X=1000$} & \multicolumn{2}{c}{ SLF } & $\theta \sim 70$ deg [4] & \multicolumn{2}{c}{ Asymptotic model [14] } \\
\cline { 2 - 7 } conditions & $c$ & $H^{*}$ & $c$ & $H^{*}$ & $c$ & $H^{*}$ \\
\hline $\bar{m}=0$ & 1.25 & 2.6 & 1.22 & 2.6 & 1.26 & 3.05 \\
0.5 & 1.21 & 2.9 & 1.2 & 2.97 & & \\
1 & 1.16 & 3.12 & & & & \\
Sticking contact & & & & & 1.21 & 3.21 \\
\hline
\end{tabular}

unloading curve decreases first, has a minimal value $\sim 1.2$ for $m_{d}=5(X \sim 10)$, then increases and attains 1.4 about for $m_{d} \sim 300(X=1000)$. Such a complex evolution is due to the evolution with $X$ of the shape of the distribution of the contact pressure $p$ [10]: this distribution becomes more and homogeneous as $X$ increases from 1 to 30 , but for higher values of $X$ whereas the mean value of $p$ is almost constant (figure 5), its value increases at the contact centre and decreases at the edge of the contact.

\section{§ 4. COMPARISON WITH OTHER APPROACHES}

\section{$\S$ 4.1. Axisymmetric approaches}

The conical indentation of RPP solids has been analysed with the asymptotic approach [14]: it considers a power law hardening rigid plastic material and neglects the material displacement; so the problem can be reduced to a flat punch equivalent problem which can be solved numerically very accurately. As the material displacement decreases for $\theta \rightarrow 90 \mathrm{deg}$ or if friction increases, we can expect that this approach provides very good results for high value of $\theta$ and/or high friction. Direct comparison with the results of the SLF approach [4] is not easy because this approach is based on Tresca yield criterion and the hardness $H$ is related to the maximal shear stress $k$. We assume $\sigma_{0}=(1+\sqrt{3} / 2) k \sim 1.85 k$ in order to recover for $\theta=90 \mathrm{deg}$ the value of the hardness provided by the asymptotic approach for zero friction: $H=3.05 \sigma_{0}$.

Table 1. Comparison of the results of present calculations for $X=1000$ and the results related to the conical indentation of RPP solids.

We see on table 1 that the results of the present calculations for the highest value of the indentation index $X=1000$ are in very good agreement with the available results of the SLF approach. On the contrary for zero friction the asymptotic approach overestimates the hardness, but provides a good estimation of the shape ratio: such a discrepancy on hardness is not surprising because the SLF approach demonstrates that for zero friction, hardness is an increasing function of the cone angle whereas it is not the case for the asymptotic approach. For sticking friction which restricts the material displacement the value of $H^{*}$ provided by the asymptotic approach is very near the value obtained with maximal friction; our value of $c$ is lower, but as the results in figure 4 suggest for $X=1000$ we have not yet attained the limiting value related to RPP solid.

Figure 7 provides the evolution of the shape ratio $c$ versus the reduced contact stiffness according to our calculations and the O\&P relation (3). Clearly the O\&P relation is correct for $m_{d}<5(X<10)$ for all contact conditions and for maximal friction it gives correct values for $m_{d}<10(X<30)$. However for higher value of the reduced contact stiffness O\&P relation underestimates the shape ratio and this induces an overestimation of the hardness. As expected this overestimation is all the more high as the indentation index is high or as the elasticity effects are small. It means that the O\&P relation works well for high elasticity material such as silica, glasses, polymers or hardened tool materials, but does not work for current metallic alloys with no significant strain hardening and strain rate hardening. 


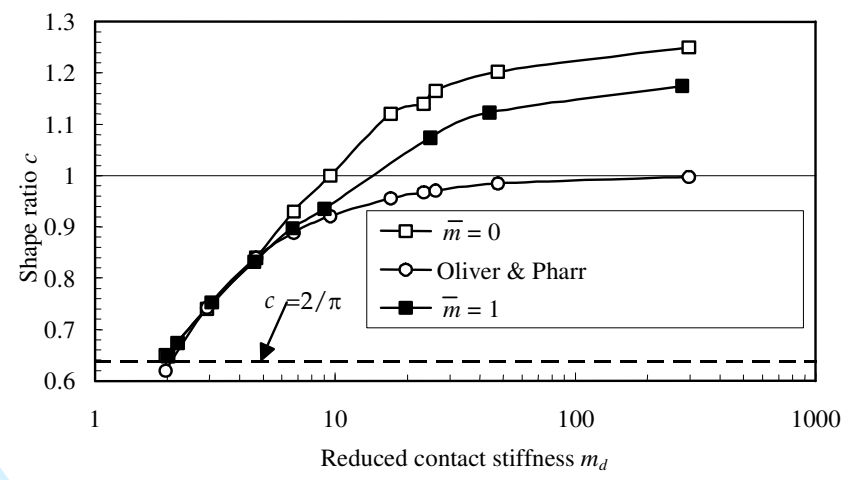

Figure 7. Evolution of the shape ratio $c$ (70.3 deg cone) versus the reduced contact stiffness $m_{d}$. For comparison the value deduced from the Oliver and Pharr relation (3) is reported.

\section{§4.3. Three-dimensional approach}
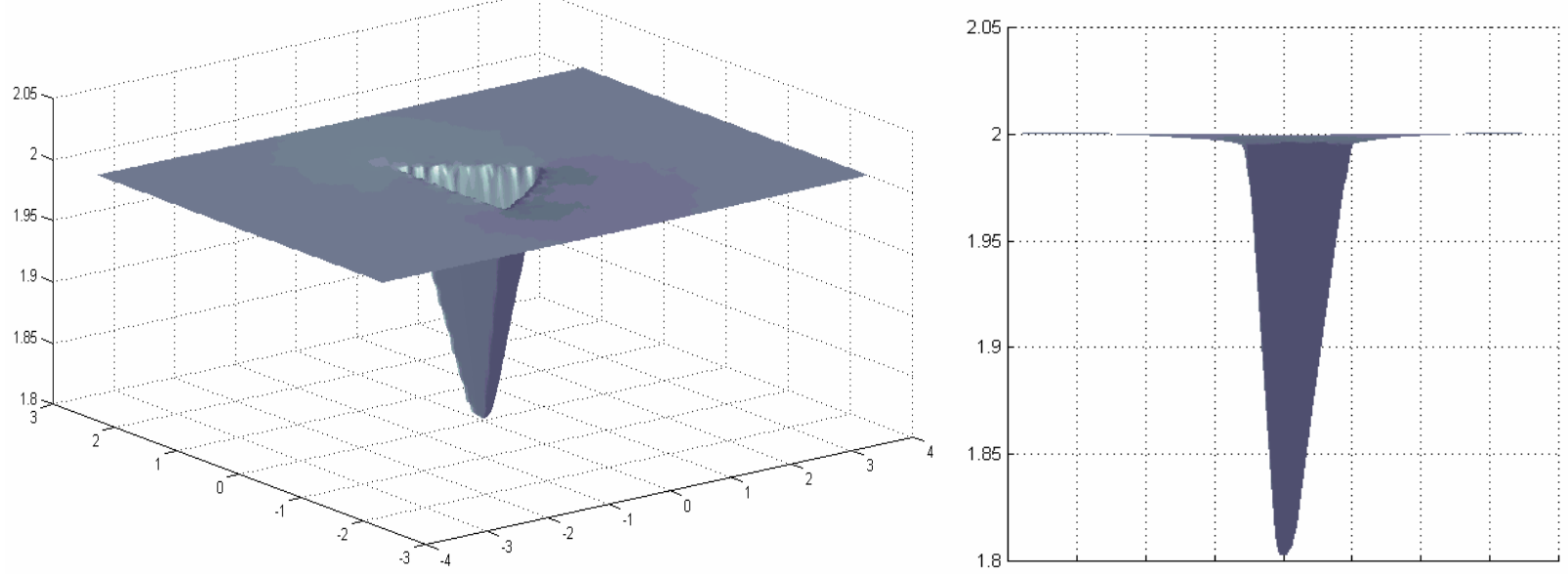

a) $X=30$ : Isometric view and section by a symmetry plane
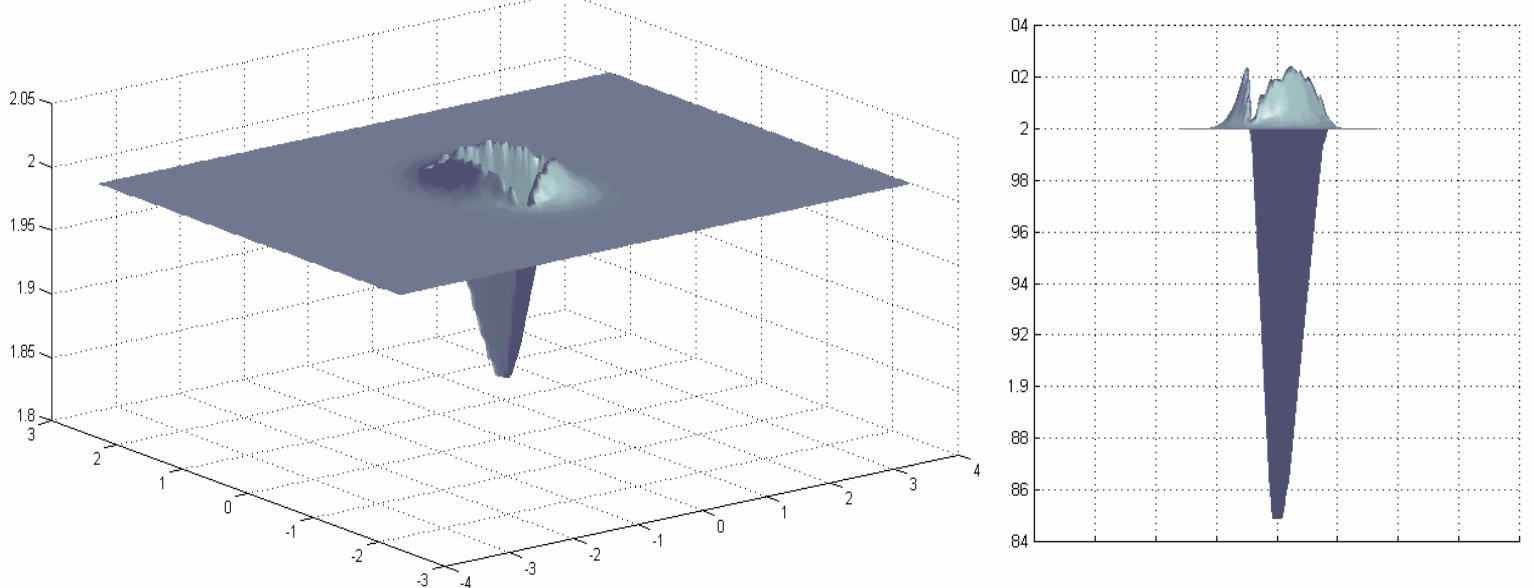

$X=100$ : Isometric view and section by a symmetry plane

Figure 8. Berkovich indent under load on EPP solids with $X=30$ and 100 (zero friction) (the unit for the axes is arbitrary). 
Figure 9. Evolution of the shape ratio $c$ for Berkovich and Vickers indentation and the equivalent axisymmetric cone versus the reduced contact stiffness $m_{d}$ (zero friction).

\section{$\S 5$. CONCLUSION}

We have analysed by the finite element method the indentation performed with the 70.3 deg cone on the elastic-perfecly plastic (EPP) solids with indentation indices $X$ ranging from 1 (quasi-elastic solid) to 1000 (quasi RPP solid). The friction shear stress is equal to zero or its maximal value. We provide so the evolution with the indentation index $X$ of the indent profile, the shape ratio $c$, the reduced hardness $H^{*}$, the reduced contact stiffness $m_{d}$ and the unloading characteristics. The influence of friction becomes significant for $X>10$ and becomes marked for high value of the indentation index: for $X=1000$ an increase in friction produces a $20 \%$ increase in hardness and a $8 \%$ decrease in the shape ratio. For $X=1000$ our results are in good agreement with the available results related to RPP solid provided by the SLF approach and the results of the asymptotic approach related to sticking friction. The results of the threedimensional numerical simulations of the Vickers and Berkovich pyramidal indentation for $X=1,30$ and 100 are in rather good agreement with the ones of the $70.3 \mathrm{deg}$ cone. The calculated evolution of $c$ with $m_{d}$ has been compared with the evolution proposed by Oliver and Pharr; this demonstrates that the O\&P relation works well for high elasticity material $(X<10-20)$ such as silica, glasses, polymers or hardened tool materials, but for current metallic alloys with no significant strain hardening and strain rate hardening it underestimates the shape ratio and so produces an overestimation of the hardness. 
APPENDIX

Results of the numerical simulations of indentation with the 70.3 deg cone of EPP solids $(X=1-5-10-20-30-60-80-100-200-1000)$

Table 2. Evolution of the shape ratio $c$ with the indentation index $X$.

\begin{tabular}{llllll}
\hline \multicolumn{7}{c}{$c=c_{0}+c_{1} \ln X$} \\
\hline$X$ & $c_{0}$ & $c_{1}$ & $X$ & $c_{0}$ & $c_{1}$ \\
\hline $1-3$ & 0.65 & 0.0273 & $1-3$ & 0.6479 & 0.0236 \\
\hline $3-100$ & 0.5183 & 0.1404 & $3-10$ & 0.5346 & 0.1307 \\
\hline $100-1000$ & 1.0039 & 0.0359 & $10-200$ & 0.5964 & 0.1022 \\
\hline & & & $200-1000$ & 1.0046 & 0.0223 \\
\hline
\end{tabular}

Table 3. Evolution of the reduced hardness $H^{*}$ with the indentation index $X$.

\begin{tabular}{llllll}
\hline \multicolumn{6}{c}{$H^{*}=H_{0}+H_{1} \ln X$} \\
\hline$X$ & $H_{0}$ & $H_{1}$ & $X$ & $H_{0}$ & $H_{1}$ \\
\hline $1-10$ & 0.5617 & 0.7298 & $1-5$ & 0.5587 & 0.7865 \\
\hline $10-30$ & 1.5973 & 0.2794 & $5-20$ & 0.8552 & 0.5893 \\
\hline $30-1000$ & 2.4824 & 0.0182 & $20-100$ & 1.9191 & 0.2415 \\
\hline \multicolumn{7}{c}{} & $100-1000$ & 2.7667 & 0.0519 \\
\hline
\end{tabular}

Table 4. Evolution of the reduced contact stiffness $m_{d}$ with the indentation index $X$.

\begin{tabular}{lllllll}
\hline & \multicolumn{5}{c}{$m_{d}=m_{0}+m_{1} X+m_{2} X^{2}$} \\
\cline { 2 - 7 } & $m_{0}$ & $m_{1}$ & $m_{2}$ & $m_{0}$ & $m_{1}$ & $m_{2}$ \\
\hline$X$ & 2.023 & -0.117 & 0.0582 & 2.023 & -0.117 & 0.0582 \\
\hline $1-5$ & 1.6 & 0.2755 & $-210^{-4}$ & 1.87 & 0.2484 & $-210^{-4}$ \\
\hline $5-200$ & -14.671 & 0.3116 & 0 & -14.858 & 0.2933 & 0 \\
\hline $200-1000$ & & & & & &
\end{tabular}

REFERENCES

[1] TABOR, D., The hardness of solids (Clarendon Press, Oxford, 1951).

[2] OLIVER, W. C., and PHARR, G. M., J. Mat. Res., 7 (6) 1564 (1992).

[3] JOHNSON, K. L., Contact mechanics (Clarendon Press, Oxford, 1985)

[4] CHITKARA, D., and BUTT, M. A., Int. J. Mech. Sci. 34 (11) 849 (1992).

[5] JOHNSON, K. L., J. Mech. Phys. Solids, 18, 115 (1970).

[6] BEC, S., GEORGES, J. M., GEORGES, E., and LOUBET, J. L., Phil. Mag. A 74 (5) 1061 (1996).

[7] DAO, M., CHOLLACOOP, N., VAN VLIET, K. J., VENKATESH, T. A., and SURESH, S., Acta Mater. 49, 3899 (2001).

[8] BUCAILlE, J. L., STAUSS, S., FELDER, E., and MICHLER, J., Acta Mater. 51, 1663 (2003). 
[9] OLIVER, W. C., and PHARR, G. M., J. Mat. Res., 19 (1) 1564 (2004).

[10] RAMOND-ANGELELIS, C., Analyse mécanique des essais d'indentation sur matériaux élastoplastiques homogènes ou multi-couches. Application à la caractérisation de la rhéologie et de la tenue mécanique des films minces, $\mathrm{PhD}$ thesis, , Ecole des Mines de Paris, France, 1998.

[11] Computer codes FORGE : http://www.transvalor.com

[12] WAGONER, R. L. and CHENOT, J.-L., Fundamentals in metal forming, (John Wiley \& Sons, New York, 1997).

[13] BHATTACHARYA, A. K., and NIX, W. D., Int. J. Solids Structures, 24 (12) 1287 (1988).

[14] BOWER, A. F., FLECK, N. A., NEEDLEMAN, A., and OGDONNA, N., Proc. $R$. Soc. Lond. A 441, 97 (1993). 